\title{
Solar Flare Effects Upon the Ionospheres of Earth and Mars
}

\author{
Michael Mendillo and Paul Withers
}

Center for Space Physics, Boston University, Boston, MA 02215

\begin{abstract}
Of the many episodic variations associated with the active Sun, perhaps the solar flare is the most spectacular and enigmatic. Because flares emit photons that reach our atmosphere in about 8 minutes, with no intervening heliospheric or magnetospheric interactions, flare effects upon the geospace system have been a somewhat neglected component in Space Weather research. Once flare photons pass the Earth, the orbit of Mars is reached in just 4 additional minutes. Recent analyses of new ionospheric data at Mars revealed electron density enhancements due to flares. On the same days, ionospheric monitoring stations on Earth equipped with University of Lowell ionosondes also recorded ionospheric flare effects. In this paper, we present observations to characterize how flares modify the ionospheres of both planets, and describe how they can be used as comparative experiments to explore how photochemical, dynamical and electro-dynamical processes operate in solar-planetary-systems.
\end{abstract}

Keywords: Ionospheres; solar flare effects, Mars' ionosphere.

PACS: $94.20 .-\mathrm{y}, 90.30 . \mathrm{gc}, 96.60 . \mathrm{qe}$

\section{INTRODUCTION}

The portion of a planet's atmosphere that experiences solar flare effects depends on the wavelengths of the photons emitted. This is the well known aspect of aeronomy that deals with the height of unit optical depth, the altitudes where photons across the wavelength spectrum are absorbed and ionize gases at those heights. For the terrestrial thermosphere, flare photons absorbed at the highest altitudes cause noticeable increases in atmospheric drag and in the total electron content (TEC) of the ionosphere. The F-layer electron density enhancements result from ionization of thermospheric oxygen (at heights $\geq \sim 150 \mathrm{~km}$ ) by increases in solar photon fluxes at EUV wavelengths $>150 \AA$ (Rishbeth and Garriott, 1969). The resultant enhancements to the overall F-layer, called sudden increases in total electron content (SITECs), were first described in detail when geostationary satellite radio beacons became available (e.g., Garriott et al., 1967, 1969; Matsoukas et al., 1972). A particularly well documented case occurred during the great solar flare of 7 August 1972 when a global network of TEC sites measured large effects (Mendillo et al., 1974). Moreover, the incoherent scatter radar (ISR) at Millstone Hill was able to record altitude profiles of changes in electron density, $\Delta \mathrm{N}_{\mathrm{e}}(\mathrm{h})$, and several plasma parameters during the same event (Mendillo and Evans, 1974).
Separate from F-layer (upper thermospheric) effects produced by EUV are the soft (20-50 $\AA$ ) and hard (1-10 $\AA)$ X-rays absorbed in the lower thermosphere and mesosphere that lead to the E-layer $(\sim 90-130 \mathrm{~km})$ and D-layer $(\sim 65-85 \mathrm{~km})$. There is a vast early literature pertaining to these studies (e.g., Donnelly, 1969), as well as a comprehensive monograph by Mitra (1974) that reviews the entire field and provides an excellent set of references. As pointed out by Mitra (1974), direct studies of the Sun were constrained to groundbased techniques (visible light and radio fluxes, in themselves not relevant to ionospheric processes) prior to the use of spacebased UV/EUV/X-ray detectors, and thus ionospheric effects caused by flares provided an important way to uncover a flare's "invisible wavelength" characteristics. The ionospheric radio science techniques celebrated by this special volume became a central component of solar physics, as well as to solarterrestrial science (e.g., Donnelly, 1971). The observational methods ranged from observations of the sudden frequency deviations (SFDs) in transmission affected by the D-layer to other RF propagation disruptions related to the E-layer and F-layer. Yet, as described by Mitra (1974), the very disruptions noted to indicate that flare effects were in progress (e.g., absorption of HF ionosonde signals) often prevented their use in ionospheric studies relating incoming photons to modified electron densities. We shall 
return to this topic later when observations on more than one planet are discussed.

Papagiannis and Matsoukas (1971) conducted the first study of simultaneous observations of flare effects upon both altitude regimes, i.e., SITECs by ionization of the thermosphere and SFDs by ionization of the mesosphere. With the coming of satellites capable of measuring the Sun's ionizing radiation directly, the need to use ionospheric proxies to obtain solar irradiance all but disappeared, but not entirely. The recent work of Thomson et al. (2004) made this point elegantly when the great flare of 4 November 2003, the largest flare ever recorded, saturated the X-ray detectors on the GOES spacecraft and the peak flux values were actually determined by their effects upon the lower ionosphere.

Elsewhere in the solar system, photons from a given solar flare can reach planets that happen to be aligned with the Sun's hemisphere of activity, with times of arrival simply related to distance, and fluxes inversely to distance-squared. The first reports of this occurring did not deal with the aeronomic consequences of an enhanced photon flux at a planet, but rather in the reflected component (albedo) of those photons. X-rays from flares in November 2003 and January 2004 were detected while making observations of Jupiter and Saturn, respectively, by Bhardwaj et al. (2005a,b). The only case to date of known flare effects upon the ionosphere of another planet comes from Mars Global Surveyor (MGS) data taken during solar flare events in April 2001 (Mendillo et al., 2006). As will be shown in the next section, to confirm such events as true flare-induced effects, the instrument most relevant to this special volume (the terrestrial ionosonde) was needed back on Earth.

\section{EXAMPLES OF IONOSPHERIC FLARE EFFECTS AT EARTH AND MARS}

\subsection{Background.}

Given the unpredictable nature of solar flares, there are two ways to achieve a successful detection of a flare's effect upon an ionosphere: continuous monitoring of the ionosphere, or very good luck with occasional observations. For observations at Earth, the global network of ionosonde stations that continuously monitors the ionosphere with a time resolution of 5-15 minutes certainly detected many cases of flare effects over the years. However, these are usually of the technique-disrupting kind (such a D-region absorption of the probing radio signal), and thus the acquisition of reliable bottomside $\mathrm{N}_{\mathrm{e}}(\mathrm{h})$ profiles of changed electron densities are far from routine. The incoherent scatter radar (ISR) technique usually transmits frequencies high enough to avoid such absorption effects, but their use is typically not in monitoring mode, but rather in short duration campaigns throughout the year, and thus luck is the key requirement for catching a flare effect. In the monograph by Mitra (1974), his Figure 112 shows ISR $\mathrm{N}_{\mathrm{e}}(\mathrm{h})$ flare profile enhancements from the ISRs at Arecibo (PR), Malvern (UK) and Millstone Hill (MA) during the solar maximum years 1967-72. These remained the definitive examples until early in this century when a series of continuous 30-day runs at ISR stations were conducted and flare effects were detected (Kudeki et al., 2005). Moreover, a new era in flare-effect studies of the terrestrial system using spacebased instruments is underway, as recently discussed by Dymond et al. (2004) and Afraimovich (2000) using ARGOS and GPS observing methods, respectively. Subsequent GPS-based studies (e.g., Tsugawa et al., 2007) have been used to confirm well known morphologies, e.g., SITEC dependence upon solar zenith angles (Mendillo et al., 1974), and ionospheric production-loss physics depending upon $\mathrm{O} / \mathrm{N}_{2}$ ratio effects (Rishbeth and Garriott, 1969).

Radio probing of the ionospheres at other planets is most often done via the radio occultation technique, and their number is exceedingly small (Schunk and Nagy, 2000). Early in the space age, Mariner, Pioneer and Voyager satellites sent to the planets were fly-by vehicles and thus only two ionospheric measurements could be made (ingress and egress) along the trajectory for each mission. Later, first at Mars and Venus, and then at Jupiter and now at Saturn, orbiting spacecraft with radio occultation experiments made more frequent observations possible. Yet for Mars, the most often measured ionosphere other than Earth's, prior to the arrival of the MGS orbiter, there were only 443 published profiles of the martian ionosphere spanning a few solar cycles (Mendillo et al., 2003). In the MGS lifetime (1998-2006) at Mars, the radio occultation experiment (Hinson et al., 1999) yielded $\sim 5600 \mathrm{~N}_{\mathrm{e}}(\mathrm{h})$ profiles measurements. The Galileo mission at Jupiter and the Cassini mission at Saturn have only a dozen or so occultation measurements in their databases, and thus the only non-terrestrial ionosphere with sufficient data to search for flare effects with a hope of confidence in detection is for Mars.

\subsection{The Flare of 7 August 1972 and its effects at Earth.}

The first week in August 1972 was one of unusually high solar and geomagnetic activity. The ISR facility at Millstone Hill was available for observations late in that week, and it caught a large 


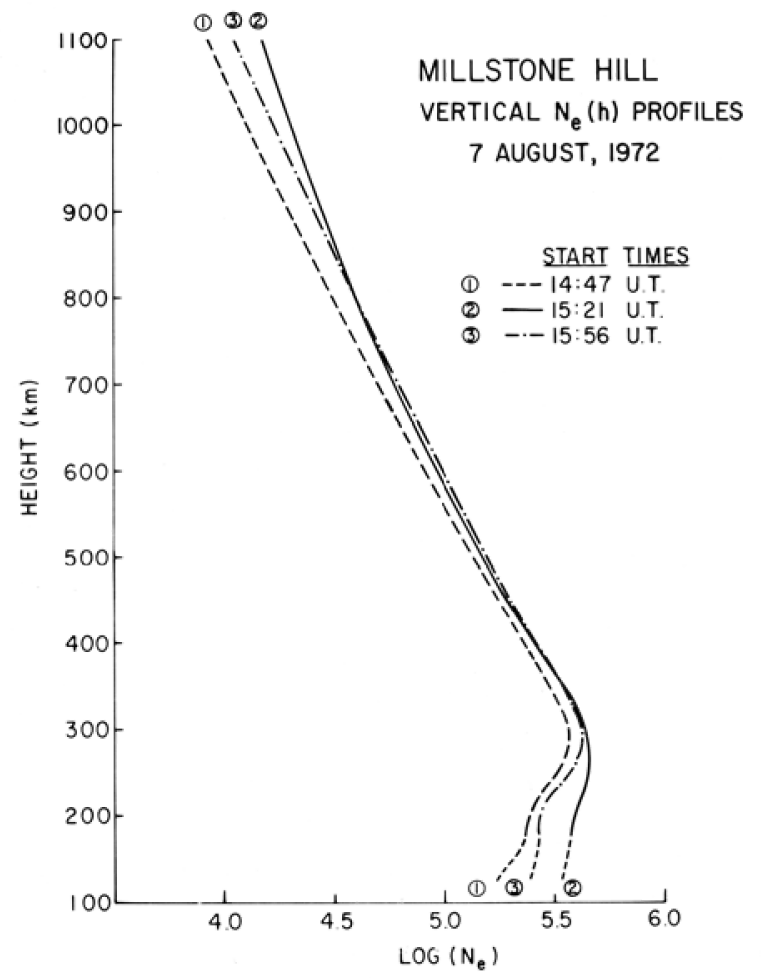

FIGURE 1. Electron density profiles (in $\log \mathrm{N}_{\mathrm{e}}$ in $\mathrm{e}^{-} / \mathrm{cm}^{3}$ ) obtained by the incoherent scatter radar at Millstone Hill (MA) during the solar flare event on 7 August 1972. While temporal resolution was limited to $\sim 35$ minutes, there is a clear change between the pre-flare and post-flare profiles. The start time of the flare in optical light was 15:00 UT, with a maximum near 15:30 UT. More relevant proxies for EUV and X-ray photons are the radio flux data shown in Fig. 5 that give the flare onset near 15:15 UT and a maximum near 15:22 UT. Percentage changes at fixed heights between profiles \#1 and \#2 are $95 \%$ at $\sim 125 \mathrm{~km}, 60 \%$ at $\sim 200 \mathrm{~km}$ and $20 \%$ at $\sim 300 \mathrm{~km}$. The integrated profiles give a total electron content increase of $\sim 4 \times 10^{12} \mathrm{e}^{-} / \mathrm{cm}^{2}$ corresponding to a $\sim 30 \%$ SITEC (see text). From Mendillo and Evans (1974).

flare effect upon the midlatitude ionosphere. Figure 1 shows three electron density profiles obtained before, during and after the time of peak flare activity. The strongest effects visible are the electron density enhancements below the F-layer peak. At the lowest altitude $(\sim 110 \mathrm{~km})$, there are soft X-ray induced ionizations, while at the peak $(\sim 300 \mathrm{~km})$ the smaller increases are due to the EUV component of the flare. That the entire profile shows an enhancement above the pre-event pattern accounts for the large sudden increases in total electron content (SITECs) observed throughout the sunlit hemisphere (Mendillo et al., 1974). One of the main messages from Fig. 1 is that both the EUV and X-ray components of the flare had to be significant, with the X-rays responsible for the enhancements at the lowest heights being dominant, as indeed appeared in modeling of this event by Sato (1975) using observed X-ray fluxes from the SOLRAD-10 satellite.

\subsection{The Flares of 15 and 26 April 2001 at Mars.}

Due to the geometry of the MGS orbit and its relationship to the Deep Space Network (DSN) on Earth, radio occultations of the spacecraft as it moves behind Mars (as seen from Earth) result in a repetition of the latitude and local time sampled. Thus, for the occultation season in April 2001 the ionospheric profiles obtained all refer to a latitude of $\sim 84^{\circ}$ North and a local time of $\sim 08: 40$ AM. On 15 April 2001 there were six such observations at 2-hour intervals, and on 26 April 2001 there were five. For such data sets, the only characteristics that changed were longitude and elapsed time between the measurements. Thus, one would expect that all of the electron density profiles would be very similar since there are no local factors (such as a global magnetic field with its dipole axis offset from the planet's axis of rotation) to make longitude sectors different.

Figure 2 shows the observations made on these two days. In panel (A), five electron density profiles are plotted in black and one in red for 15 April; four in black and one in red are plotted for 26 April. The red curves are the only ones that show significant departures from the means of the other observations on those days. The percentage differences at each altitude from the means of the control curves are shown in panel (B). There are a few characteristics to notice. First of all, Mars has an ionosphere that spans a small range in altitudes, with a main peak near $130 \mathrm{~km}$ (produced by EUV) and a secondary peak near $110 \mathrm{~km}$ (produced by soft X-rays); both layers are in photochemical equilibrium and therefore respond predictably to increases in solar activity (Rishbeth and Mendillo, 2004). There is a marked inconsistency of only one profile, and it is confined to heights below the altitude of peak density on each day. Figure 3 shows the reasons for these non-typical bottomside profiles. The GOES satellite in earth-orbit detected solar flare X-ray bursts shortly before the MGS measurements were made. The arrows indicate when the X-rays detected at Earth would reach Mars at the times of the MGS observations, and they fall $\sim 20$ minutes and $\sim 90$ seconds after the peak fluxes at Mars. Mars was near the configuration called opposition in early 2001, meaning it was nearly aligned with the Sun and Earth, and therefore experiencing the same flare irradiance but, due to its distance of $\sim 1.5 \mathrm{AU}$, a flux reduced by a factor of 2.25 and an arrival time $\sim 4$ minutes later that the 8 minute travel time from the 

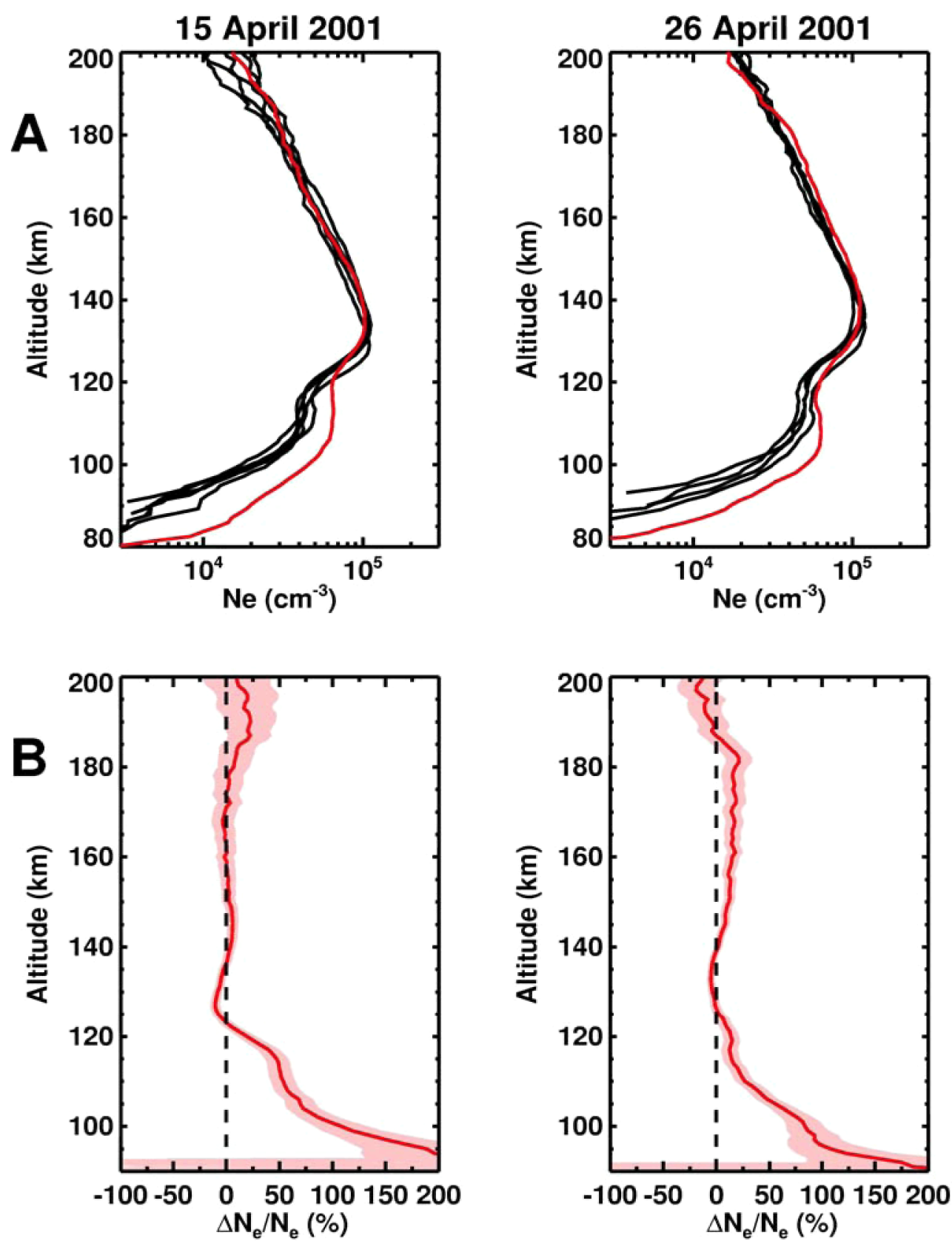

FIGURE 2. (A) Electron density profiles at Mars obtained by the Mars Global Surveyor (MGS) radio occultation experiment on 15 and 26 April 2001, days of solar flares. Measurement uncertainty is several thousand $\mathrm{e}^{-} / \mathrm{cm}^{3}$, and thus statistically significant departures at low altitudes occur in one profile on each day (shown in red, corresponding in time to 14:15 UT and 13:16 UT, respectively). (B) Percentage differences between the flare-affected profiles and the averages of the other profiles. The shadings give the $1-\sigma$ standard error in the relative change in $\mathrm{N}_{\mathrm{e}}$ at each height. From Mendillo et al. (2006).

Sun to the Earth. While the two flares were very different in peak emission, the fact that they had comparable flux values at the times of the observations must account for the very similar magnitudes of the electron density changes seen in the MGS profiles at those times.
The attribution of the effects at Mars in Fig. 2 to the flare characteristics observed at Earth in Fig. 3 can be made more robust if flare effects could be seen at Earth as well. To do this, we turn to the network of ionosondes built by the University of Lowell under the direction of Professor Bodo Reinisch and select 

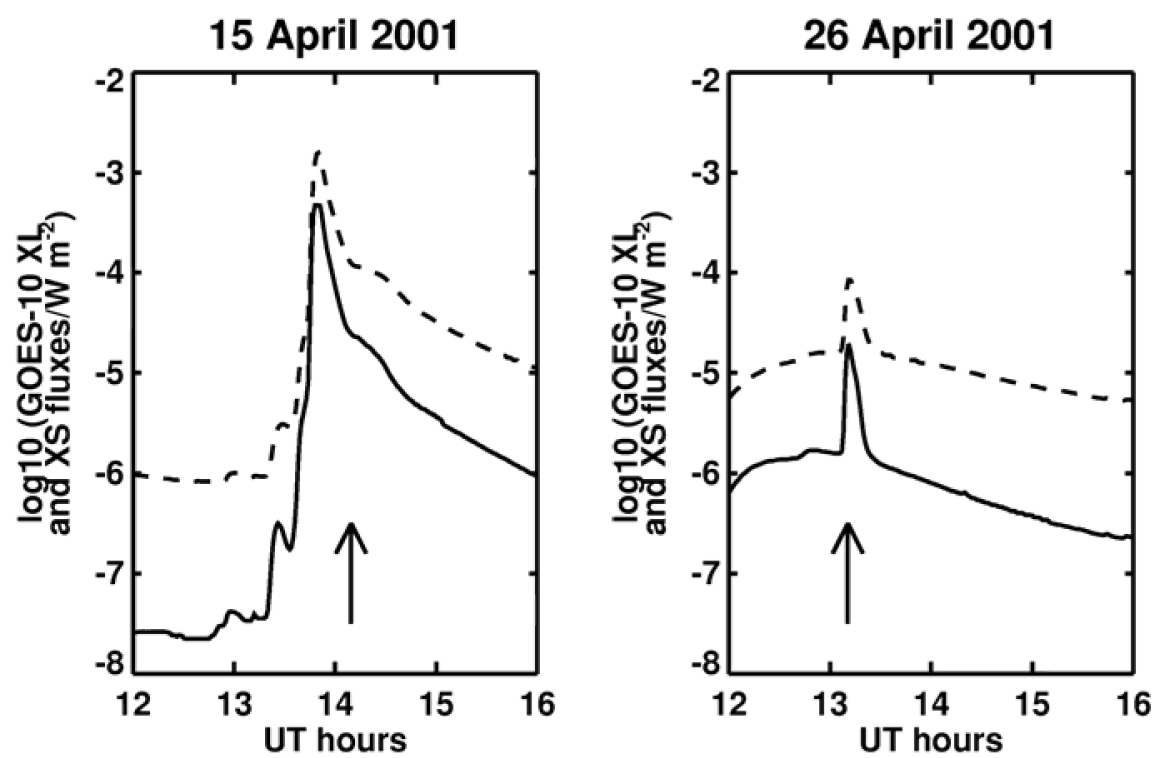

FIGURE 3. Solar X-ray fluxes on 15 and 26 April 2001 measured by the GOES spacecraft at Earth for two wavelength bands: XS $(0.5$ to $3 \AA$ ), solid line, and XL ( 1 to $8 \AA$ ), dashed line. The flares were classified as X14.4 and M7.8, and the peak fluxes at Earth occurred at 13:50 UT and 13:10 UT, respectively. The solar fluxes incident upon Mars at the times of the flare-affected profiles in Fig. 2 are marked by arrows. From Mendillo et al. (2006).

several stations that happened to be in the dayside hemisphere at Earth. Data from these sites are shown in Fig. 4. Since the lower peak of the martian ionosphere is caused by soft $\mathrm{X}$-rays, as is the case for the terrestrial E-layer, we examined the peak electron density of that layer, as recorded at stations in the UK (Chilton), the USA (Millstone Hill and Wallops Island), and in Greenland (where the solar zenith angle was comparable to the MGS high latitude measurement sites on Mars). The scalings of the critical frequency of the E-layer (foE) from the ionograms at each station were done personally by Professor Reinisch, his first use of ionosonde data to help understand the ionosphere at Mars.

The data in Fig. 4 show, in many ways, the classic examples of the ionospheric (and an ionosonde's) response to a solar flare. For the strong X-ray event on 15 April 2001, there is a loss of ionosonde data due to D-region absorption at all of the stations. The duration of missing data is shortest at Chilton, much longer at the two US east coast stations, and for the remainder of the day in Greenland. The GOES data refer to hard $\mathrm{X}$-rays, ones that would ionize regions below the Elayer (and below the secondary layer on Mars as well), and thus this is consistent with well known solarterrestrial physics. For the less intense flare of 26 April 2001, there is no loss of ionosonde data and increases in ionospheric densities at E-layer heights were observed at all of the stations. These are due to the softer X-rays in that flare, the same portion of the solar irradiance responsible for the effects noted at
Mars. Thus, the Lowell ionosonde network played a central role in validating conclusions reached more tentatively using only MGS data at Mars. The value of continuous measurements is demonstrated as well and. as discussed next, the role terrestrial ionosondes can play in comparative aeronomy.

\section{MODELING ISSUES}

A full treatment of modeling flare effects upon a planet's ionosphere is clearly beyond the scope of this paper. Indeed, it is an area of active research for terrestrial space physics, and barely underway for planetary atmospheres. The fundamental value of such work deals with the role of a solar flare as perturbation experiment. When a sudden change occurs in a single parameter in a multi-process system, attempts at modeling the response serve as a test of our knowledge of all of the processes in a highly coupled system. To get the pre-flare ionosphere modeled correctly, the processes of solar production, ionospheric loss and plasma transport need to be handled properly, as described for the terrestrial case by Sato (1975) and most recently by Huba et al. (2005). Baseline models for the martian ionosphere appear in Fox et al., (1996), Krasnopolsky (2002) and Martinis et al. (2003). At the time of the solar flare, only the irradiance changes abruptly, and thus it is tempting to think that only solar ionization processes change. Yet, the neutral atmosphere responds to changes in solar flux as well 

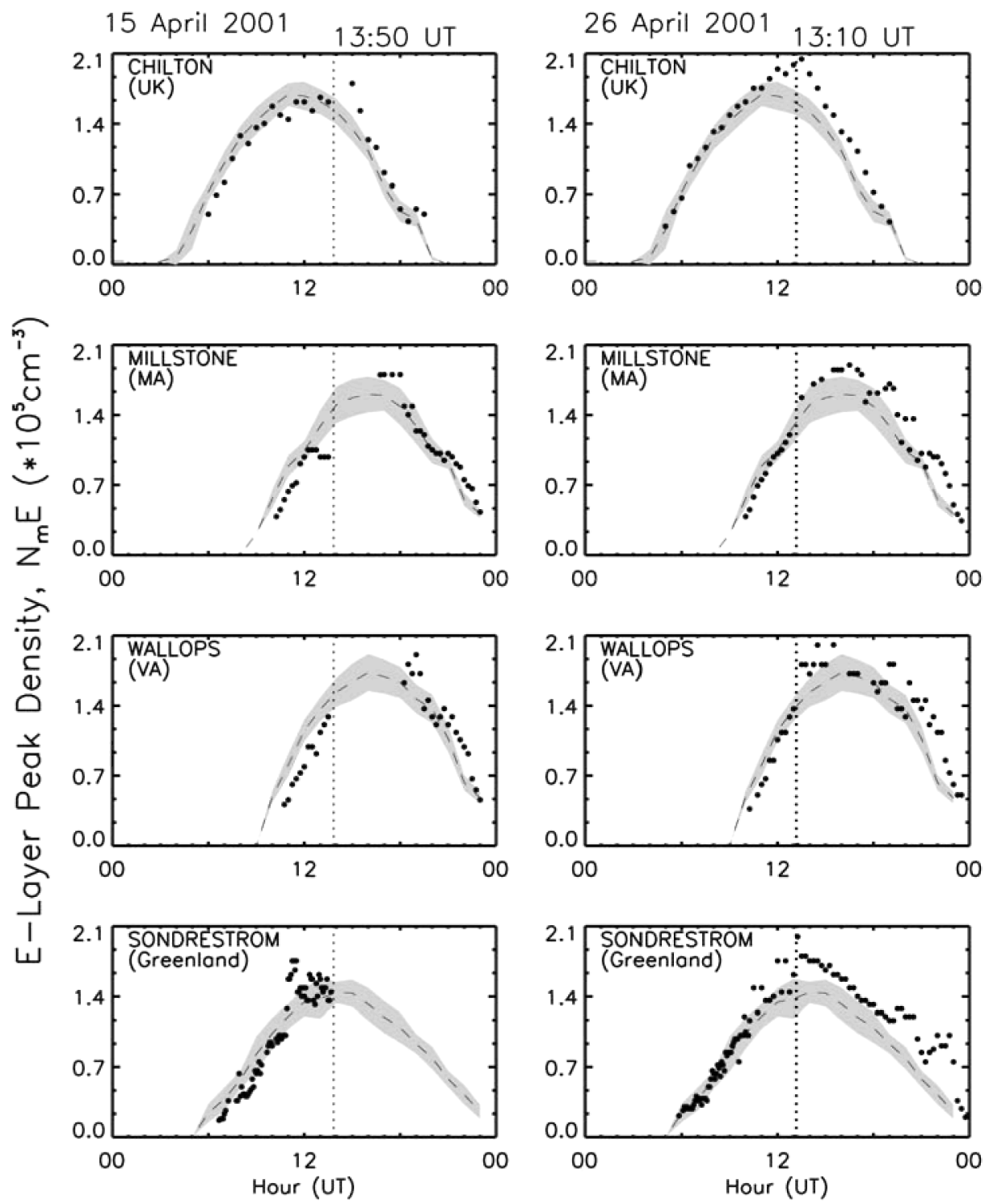

FIGURE 4. The maximum electron density of the Earth's ionospheric E-layer $\left(\mathrm{N}_{\mathrm{m}} \mathrm{E}\right)$ for 15 and 26 April 2001, as measured by University of Lowell ionosondes at several sites in the sunlit hemisphere. For each station, the monthly mean pattern is given by the dashed lines, their standard deviations $(1-\sigma)$ by the shading, and individual data points by dots (scaled to $0.1 \mathrm{MHz}$, giving $\sim 10 \%$ uncertainty). The vertical dotted lines show the times of peak flare fluxes. At the highest latitude station (Sondrestrom, Greenland), there is additional preflare E-layer variability on 26 April caused by sporadic-E layers and by ionizations from precipitating auroral electrons. From Mendillo et al. (2006).

play. While photochemical time scales are fast, so are electrodynamical ones. Thus, changes to ionospheric conductivities occur and the ionosphere responds quickly to them.

Early attempts to treat all of these processes involved a great many assumptions about mechanisms we now can document more completely. Thus, Sato (1975) had to assume how the solar irradiance could be scaled using hard X-rays and radio fluxes as proxies. Figure 5 gives an example of such a correlation, using the same great flare event of 7 August 1972. Clearly, the time rate of change of TEC is related to the temporal evolution of the flare as seen in $0.857 \mathrm{~cm}$ radio observations, even though a 35,000 $\mathrm{MHz}$ photon has no effect upon ionospheric production. While an impressive proxy heuristically for solar production, a simple scaling of a pre-event solar irradiance by a radio flux pattern (e.g., using 
F10.7) ignores the fact that the characteristics of irradiance change during a flare as well. That is, the increase of soft X-rays is not the same multiplicative factor as seen at EUV or UV wavelengths. Today,

\section{RATE OF IONOSPHERIC ELECTRON CONTENT CHANGE \\ 7 AUGUST 1972}

( UNITS OF ELECTRONS - METER ${ }^{-2}-\mathrm{SEC}^{-1} \times 10^{-14}$ )
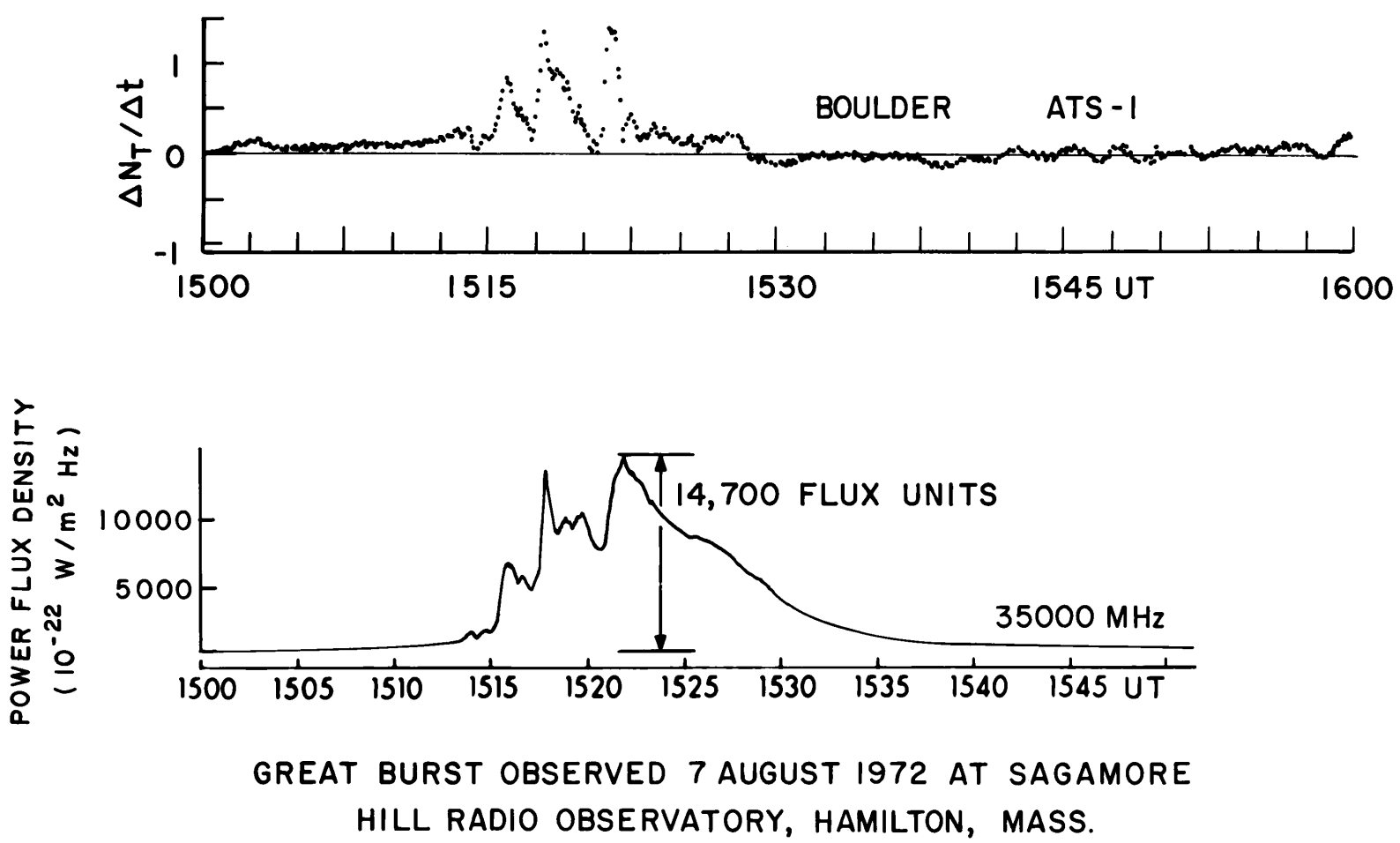

FIGURE 5. High time resolution measurements of (top) ionosphere total electron content rate of change and (bottom) solar radio flux at 35,000 MHz during the flare event on 7 August 1972. The TEC data were observed from a station in Boulder (CO), and the radio flux from the AFCRL Sagamore Hill Observatory in Hamilton (MA). The strong correlation results from the ionospheric continuity equation that relates temporal changes in ionospheric densities to a solar production function (Rishbeth and Garriott, 1969). Previous studies had shown that the 35,000 MHz radio flux is an excellent proxy for solar EUV and X-ray burst characteristics (Castelli and Richards, 1971; Matsoukas et al., 1972). Taken from Mendillo et al. (1974).

we have a new model for solar irradiance based on actual measurements over the full spectrum of interest in aeronomy (Tobiska, 2000; 2004), with the first treatment of flare events to appear soon (Chamberlin, P., private communication, 2007). Thus, modeling of a solar induced effect can now be done without making a remarkably strong assumption about the magnitude and time profile of the photons of interest. For example, Huba et al. (2005) used a background 37-segment spectral model with a time-dependent flare sub-model in successful simulations of the SITEC magnitudes observed during the great flare of 14 July 2000 .

Increases in solar input heat an atmosphere and the subsequent thermal expansion changes the mix of constituents at fixed heights. This effect acts at all planets, but with very different efficiencies, as recently shown for the first time in studies of solar rotation driven atmospheric expansion effects at Venus, Earth and Mars by Forbes et al. (2006). For a flare, the rise time to peak emission is often very short ( $\sim$ minutes), less than thermospheric time constants for heating and thermal expansion, and thus the neutral atmosphere used to model the pre-flare ionosphere may be used during the flare. This is not the case for other time scales of variability (diurnal, seasonal, solar cycle), and thus flares are indeed "controlled experiments" that change only one parameter (solar irradiance), and thus are capable of addressing details of the photochemical system.

\section{COMPERATIVE AERONOMY OF SOLAR FLARE EFFECTS}


Prior to the MGS observations of flare effects at Mars, all studies of flare-induced processes within an atmosphere dealt with terrestrial systems. While significant progress has been achieved in understanding our atmosphere's response to a solar flare, it remains true that a comprehensive understanding of a physical process requires verification and validation at other venues. Comparative planetary studies offer opportunities to study "system-response-functions" of a given input at multiple locations in the solar system. There are several aspects that deal with flares that serve as excellent examples of how comparative studies can enhance our overall understanding of solar-planetaryrelations.

Here we discuss three such cases: Secondary ionization, magnetic crochets, and atmospheric escape.

\subsection{Secondary Ionization.}

Since flare effects are predominantly a high energy photon phenomenon, the issue of secondary ionization is a critical factor to simulate properly in models. Each soft X-ray photon produces far more than a single ion-electron pair, and thus this secondary ionization factor becomes crucial. To date, the constraints on secondary ionization arise from attempts to model ambient morphologies (diurnal, seasonal, solar cycle), and thus the uncertainties in other factors (e.g., neutral winds, electric fields) are larger. Thus, while attempts to "tune" agreement between models and data using secondary ionization as the free parameter can be tried for photochemical layers (Moore et al., 2004), the correction factors involved are really the sum of all uncertainties in all of the processes acting at that time. With a solar flare, there is clearly a pulse in production and, in the early time response, constraints on secondary ionization have to play a far more central role. Thus, flares become active experiments in basic photochemistry. The parameterization of secondary ionization used for preflare simulations must remain unchanged for flare simulations, and this removes the ability for an arbitrary tuning of this important factor. For Earth, the species of interest are $\mathrm{N}_{2}, \mathrm{O}_{2}$ and $\mathrm{O}$ and their secondary ionization have been studied (e.g., Lilensten et al., 1989) and parameterized by Richards and Torr (1988) and Titheridge $(1996,2000)$. In the terrestrial SITEC model study described by Huba et al. (2005), the discrepancy in fine scale temporal agreement between simulations and data was attributed to such poorly constrained effects.

On Mars, $\mathrm{N}_{2}$ and $\mathrm{O}$ are minor constituents, and thus secondary ionization comes primarily from the photo-ionization of the far more abundant $\mathrm{CO}_{2}$. This has been treated in studies by Fox et al. (1996) and Fox (2004a,b). A solar flare that causes ionospheric enhancements on two planets can thus be used as a 'laboratory-in-space' experiment, linking observations and models, to constrain the secondary ionization process for $\mathrm{N}_{2}, \mathrm{O}_{2}, \mathrm{O}$ and $\mathrm{CO}_{2}$.

\subsection{The Magnetic Signature of a Flare.}

The beginnings of solar-terrestrial physics are most often associated with speculations about the origins of the aurora and, in particular, their correlations with sunspot activity (Eather, 1980). An aspect of this history relevant to the topic at hand is the first recorded observations of a solar flare. That occurred in England at 11:18 GMT on 1 September 1859 while R. C. Carrington (1859) and R. Hodgson (1859) were simultaneously, and independently, making observations of sunspots. They reported their results at the November meeting of the Royal Astronomical Society (RAS) and were shown records of the geomagnetic field as measured in Kew that exhibited "a moderate but very marked disturbance." In the RAS proceedings of that meeting, the recorded discussion noted that "While the contemporary occurrence may deserve noting, he (Mr. Carrington) would not have it supposed that he even leans towards hastily connecting them. 'One swallow does not make a summer'." Seven solar cycles later, J. A. Fleming (1936) reported that a fade-out of ionospherically reflected radio waves occurred simultaneously with a "sun-spot eruption" and a 70-gamma change in the horizontal component of the geomagnetic field. Since the event was not observed at stations in the night hemisphere, Fleming correctly argued that the radio effect was probably due to absorption caused by solar radiation which increased the ionization in the regions of high collision frequency. Fleming further noted that the lack of a deflection in the vertical component suggested that the magnetic variations could be described by an overhead horizontal current.

Figure 6 gives examples of the sudden flare effect (SFE) at several stations in Europe and in North America during the 7 August 1972 flare. The direction and magnitude of the deflection of the H-component is obviously not the same in different local time and latitude regions. In one of the oddest uses of terminology in space physics, this effect is called a magnetic crochet (probably related to the French word for "hook" or "loop" to describe the departure and return of the magnetometer tracing to pre-flare conditions). The explanation given by Fleming (1936), Ratcliffe (1960), and others is that the sudden increase in ionization caused by the flare adds 
electrical conductivities at heights where wind-induced dynamo action causes current to flow. If the ionization
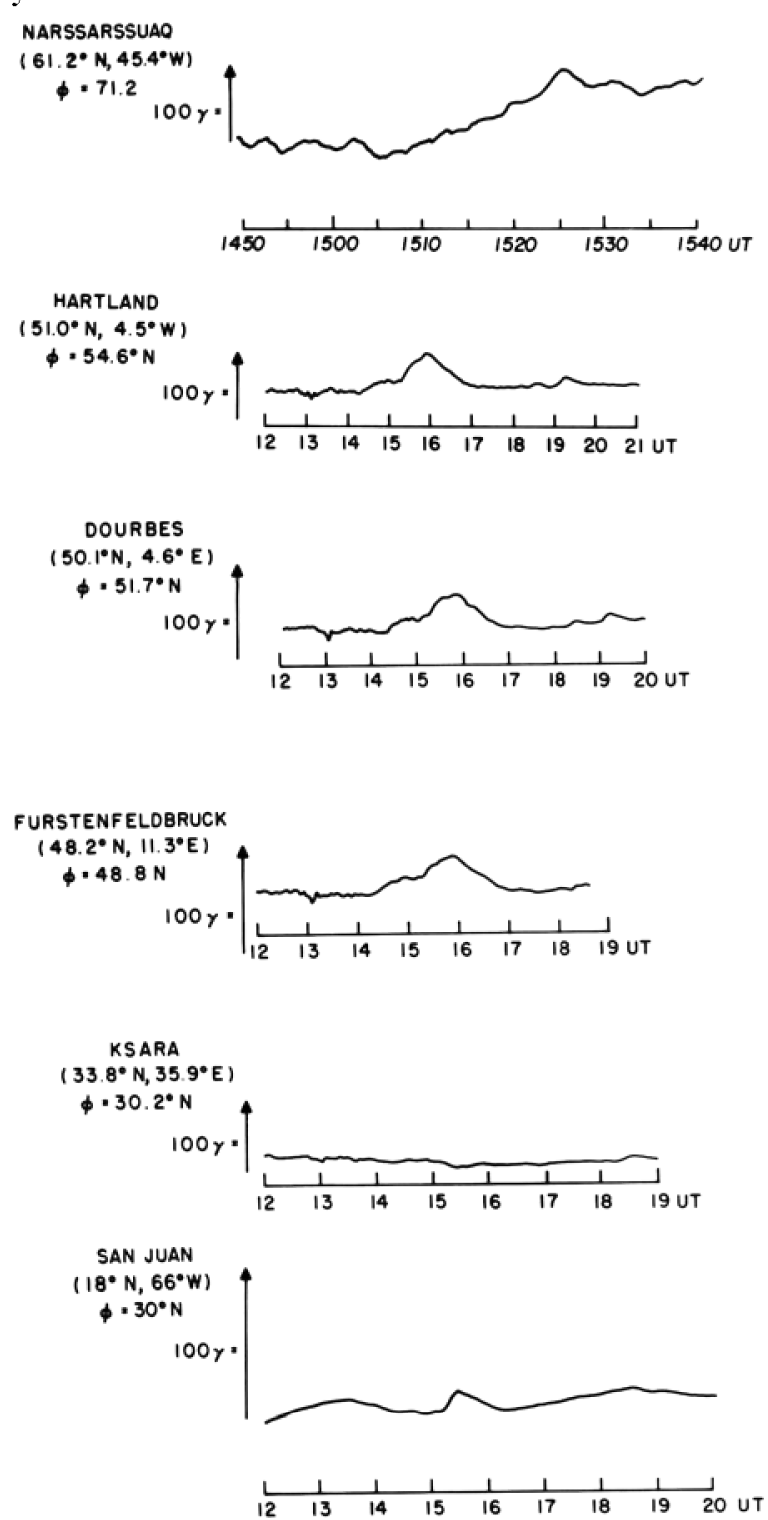

is produced at a height where the daily dynamo currents flow, then the crochet deflection is in the
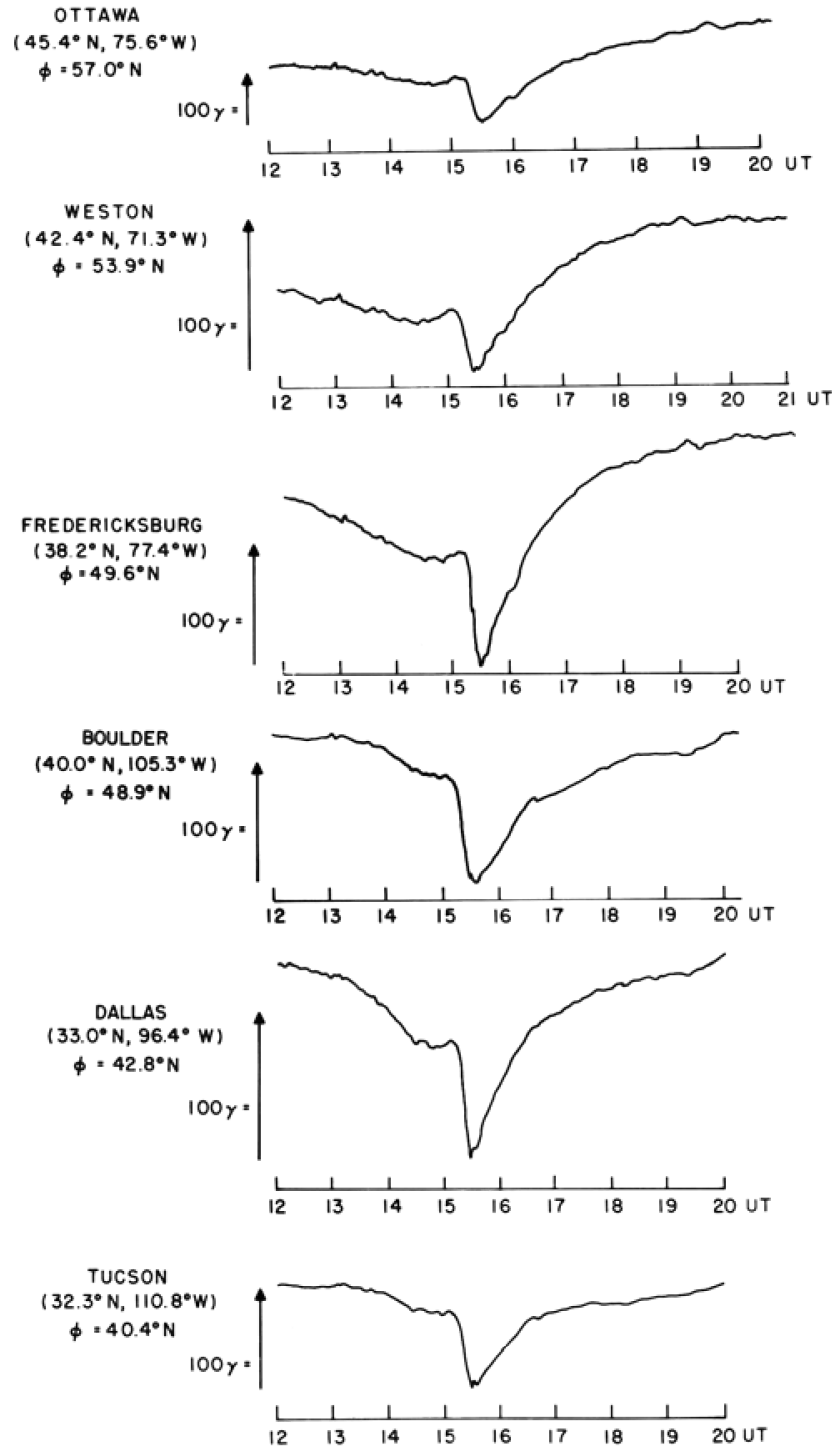

FIGURE 6. Examples of sudden flare effects (SFEs), also called magnetic crochets, during the flare of 7 August 1972 . Stations from two longitude sectors (European and North American) are shown to illustrate that difference in local time contribute to the type of magnetic variation seen (see text). Taken from Mendillo et al. (1974).

same direction. If the ionization produces the conductivity enhancement at a different height, where the winds might be in a different direction, then the magnetic deflection would be changed. The crochet phenomenon was thus a diagnostic of E-layer and/or mesospheric winds, as well as shears in those winds (Richmond and Venkateswaran (1971). Could this effect occur on Mars?

It had long been known that Mars does not have a global magnetic field, and thus that the solar wind interaction was one that did not produce a magnetosphere, but rather a comet-like ionopause and iono-tail. The MGS spacecraft carried a magnetometer to study such effects; the surprising result was that regions of magnetic flux associated with the planet were discovered. These localized regions were termed crustal fields to portray their nonglobal characteristic and association with sub-surface sources at various places on the planet, perhaps as remnants of a once global dipole. Figure 7 gives a 
model representation of those fields at longitudes in Mars' southern hemisphere where they are most

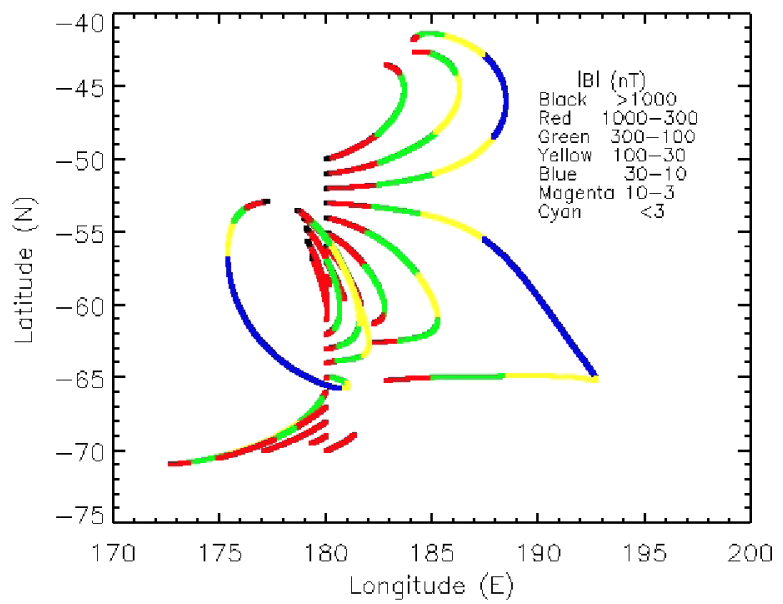

prominent. Note that within relatively short distances, the field topology changes from equatorial-like

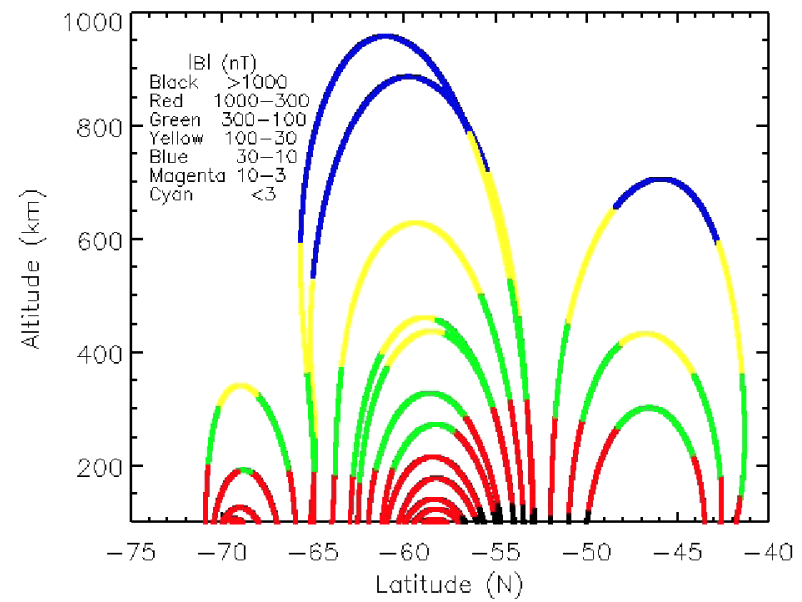

FIGURE 7. A model representation (Arkani-Hamed, 2004) of the crustal magnetic fields discovered at Mars by the magnetometer onboard the MGS spacecraft (Acuña et al., 2001), showing field strength on grids of latitude and longitude (left), and height and latitude (right) at longitude 180 (E). In this longitude sector in Mars' southern hemisphere, a series of subsurface dipole-like sources result in transitions from equatorial to polar magnetic topologies over spatial distances far smaller than the size of the planet's radius. Flare-induced enhancements to ionospheric conductivities in such a region could cause marked changes in ambient wind-driven dynamo current effects upon local magnetic fields, effectively creating SFE/crochet effects at Mars (see text).

\section{MILLSTONE HILL TEMPERATURE AND VELOCITY PROFILES - - - 7 AUGUST, I972}

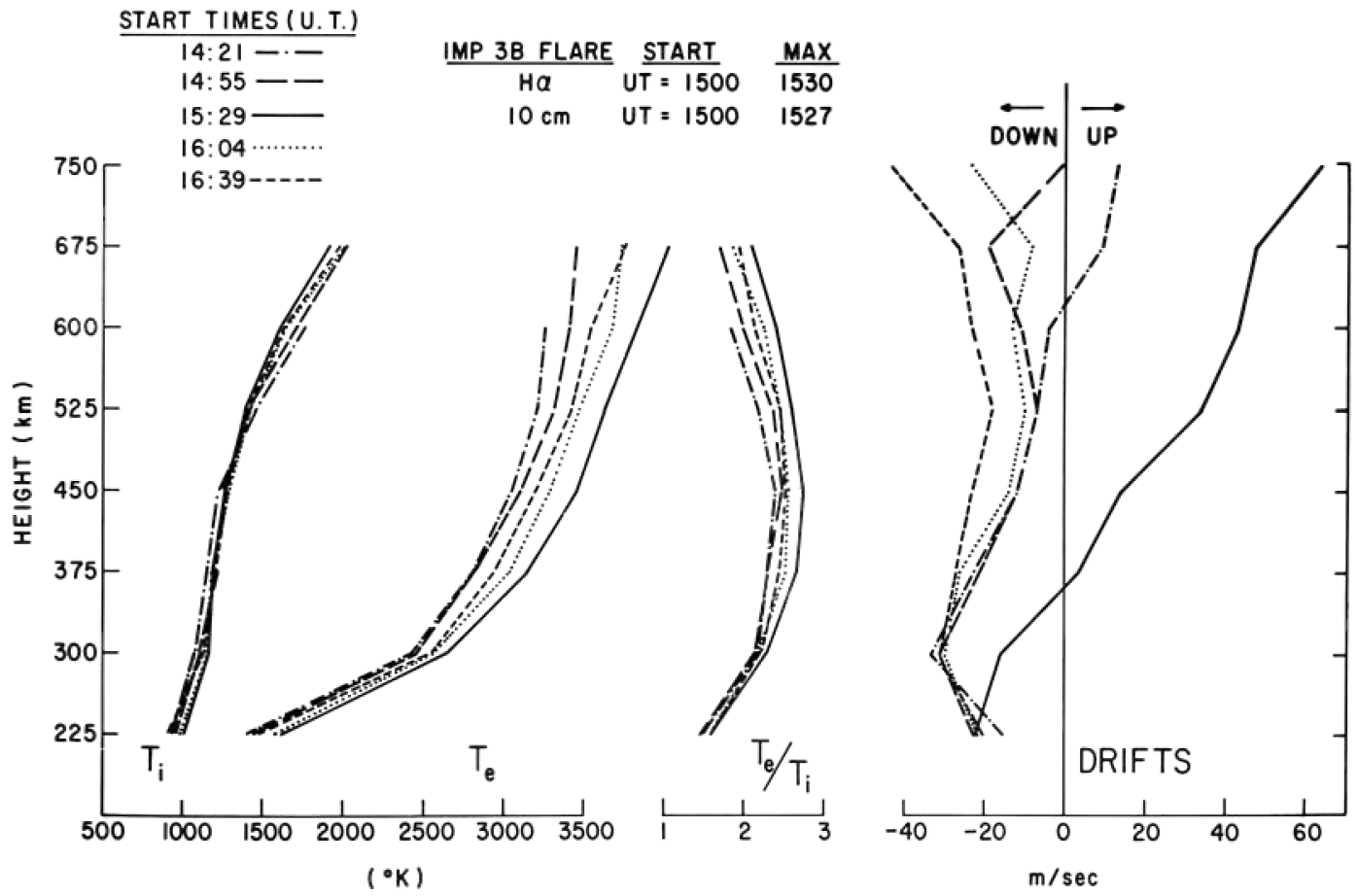

FIGURE 8. Ionospheric plasma parameters measured by the incoherent scatter radar at Millstone Hill during the flare of 7 August 1972. Altitude profiles of ion $\left(\mathrm{O}^{+}\right)$temperature $\left(\mathrm{T}_{i}\right)$, electron temperature $\left(\mathrm{T}_{e}\right)$, the $\mathrm{T}_{e} / \mathrm{T}_{\mathrm{i}}$ ratio, and vertical plasma drift are given at times before, during and after the flare. Note that the profiles at 15:29 UT (solid lines) show increases in $\mathrm{T}_{\mathrm{e}}$ and in 
upward plasma drift. The thermally-driven upward drift alters the shape of the flare-affected profile in Fig. 1, suggesting that similar flare effects at Mars might have contributed to its atmospheric escape (see text). From Mendillo and Evans (1974).

(horizontal B) to cusp-like (vertical B). Winds in these regions would thus affect ionospheric plasma in very different ways, possibly including dynamo action, as discussed briefly in Mendillo et al. (2003). Flare induced augmentations to the ionosphere, both in its regular layers, as well as in lower regions, would thus add conductivities and produce dynamo action that might lead to observable changes in the magnetic fields. When magnetometers are placed on the surface of Mars, flare-induced martian crochets may well be used to help understand the upper atmospheric wind patterns on Mars.

\subsection{Ionospheric Escape.}

One of the main topics of atmospheric research at Mars is the loss of its previously dense atmosphere. Could solar flares have contributed to loss over the history of the planet? The two electron density profiles with obvious flare effects shown in Fig. 2 do not suggest in themselves that the ionization produced escaped. The $\mathrm{N}_{\mathrm{e}}$ enhancements were confined to the bottomside ionosphere where the neutral atmosphere is dense, and thus transport slow in comparison to chemical recombination time scales. In addition, these two profiles do not show changes in the slopes of the topside ionosphere, again indicating that upward flow is not at work. Yet, the two profiles do not come from a continuous monitoring of the ionosphere at Mars, and thus time histories of flare effects might show both topside enhancements and signs of upward diffusion. Indeed, the radar on the Mars Express mission (essentially a topside ionosonde) recorded a flare effect on 15 September 2005 with a strong enhancement in peak densities (Gurnett et al., 2005). Once flare-created plasma reaches altitudes above $\sim 300 \mathrm{~km}$ at Mars, it is subject to capture by the solar wind, and thus atmospheric constituents can be lost from the planet.

At Earth, upward diffusion of flare-produced plasma is not considered an escape processes of importance since most of the dense ionosphere is at mid-to-low latitudes where upward diffusion would be to the plasmasphere, i.e., to regions of closed magnetic field lines and thus flux tubes of plasma that essentially co-rotate with the Earth. The flare-affected profile in Fig. 1 shows a change in slope (scale height) well above the peak ( $h>600 \mathrm{~km})$, indicative that the flare indeed provoked vertical motions. One of the benefits of the ISR technique is that plasma temperatures and drifts can be measured simultaneously with the electron densities, and Fig. 8 shows the plasma parameters measured at Millstone on
7 August 1972. The profile obtained during peak flare activity (solid line) shows increases in electron temperature (presumably from the burst in photoelectrons produced by the enhanced flux of EUV absorption in the F-layer), and the dramatic surge in vertical drifts they caused (Mendillo and Evans, 1974). Without a magnetosphere to retain such plasma, similar effects at Mars would lead to escape

\section{ACKNOWLEDGEMENTS}

This work was supported by a grant from NASA's Mars Data Analysis Program (MDAP), and from the comparative studies component of a grant from the NSF Aeronomy Program. The Mars Global Surveyor $\mathrm{N}_{\mathrm{e}}(\mathrm{h})$ profiles were provided by Dr. David Hinson, and the Lowell ionosonde data were provided by Professor Bodo Reinisch. Ms. Clara Narvaez helped with the manuscript preparation.

\section{REFERENCES}

1 M. H. Acuña et al., Magnetic field of Mars: Summary of results from the aerobraking and mapping orbits, J. Geophys. Res., 106, 23, 40323,418 (2001).

2. E. L. Afraimovich, GPS global detection of the ionospheric response to Solar flares, Radio Sci., 35(6), 1417-1424 (2000).

3. J. Arkani-Hamed, A coherent model of the crustal magnetic field of Mars, J. Geophys. Res., 109. E09005, doi: 10.1029/2004JE002265 (2004).

4. A. Bhardwaj, G. Branduardi-Raymont, R. F. Elsner, G. R. Gladstone, G. Ramsay, P, Rodrignez, R. Soria, J.H. Waite, Jr., and T. E. Cravens, Solar control of Jupiter's equatorial Xray emissions: 26-29 November 2003 XMMNewton observations, Geophys. Res. Lett., 32, L03S08, doi:10.1029/2004GL021497 (2005a).

5 A. Bhardwaj, R. F. Elsner, J. Huner Waite, Jr., G. R. Gladstone, R. E. Cravens, and P. G. Ford, Chandra observations of an X-ray flare at Saturn: Evidence of direct solar control on Saturn's disk X-ray emissions, Astrophys. J., 624:L121-L124, (2005b).

6 R.C. Carrington, Description of a singular appearance seen in the Sun on September 1, 1859 , Mon. Not. Roy. Astron. Soc., 30, 13-15 (1859).

7 J. P Castelli and D. W. Richards, Observations of solar bursts at microwave and extreme ultraviolet wavelengths, J. Geophys. Res., 76, 8409-8413, (1971). 
8 R. F. Donnelly, Contribution of X-ray and EUV bursts of solar flares to sudden frequency deviations, J. Geophys. Res., 74, 1873-1877, (1969).

9 R. F. Donnelly, Extreme ultraviolet flashes of solar flares observed via sudden frequency deviations: experimental results, Solar Phys., 20, 188-203 (1971).

10 K. F. Dymond et al. (7 co-authors), Ionospheric response to the solar flare of 14 July 2000 , Radio Sci., 39, RS1S25, doi:1029/2002RS002842 (2004).

11 R. Eather, Majestic Lights: The Aurora in Science, History, and the Arts, American Geophysical Union, Washington, DC, 1980.

12 J. A. Fleming, Notes on radio fade-out of August 25, 1936, Terr. Mag. Atmosph. Elec., 41, 404-406 (1936).

13 J. M. Forbes, S. Bruinsma, and F. G. Lemoine, Solar rotation effects in the thermospheres of Mars and Earth, Science, 312, 1366-1368 (2006).

14 J. L. Fox, P. Zhou, and S. W. Bougher, The martian thermosphere/ionosphere at high and low solar activities, Adv. Space Res., 17(11), 203-218, (1996).

15 J. L. Fox, Advances in the aeronomy of Venus and Mars, Adv. Space Res., 33, 132-139 (2004a).

16 J. L. Fox, Response of the Martian thermosphere/ionosphere to enhanced fluxes of solar soft X rays, J. Geophys. Res., 109, A11310, doi:10.1029/2004JA010380 (2004b).

17 O. K. Garriott, A. V. da Rosa, M. J. Davis, and O. G. Villard, Solar flare effects in the ionosphere, $J$. Geophys. Res., 72, 6099-6103 (1967).

18 O. K. Garriott, A. V. da Rosa, L. A. Wagner, and G. D. Thome, Enhancement of ionizing radiation during a solar flare, Solar Phys., 8, 226-239 (1969).

19 D. A. Gurnett, D. L. Kirchner, R. L. Huff, D. D. Morgan, A. M. Persoon, T. F. Averkamp, F. Duru, E. Nielsen, A. Safaeinili, J. J. Plaut, G. Picardi, Science, 310, 1929-1933 (2005).

20 D. P. Hinson, R. A. Simpson, J. D. Twicken, and G. L. Tyler, Initial results from radio occultation measurements with Mars Global Surveyor, J. Geophys. Res., 104, 26997-27012 (1999).

21 R. Hodgson, On a curious appearance seen in the Sun, Mon. Not, Roy. Astron. Soc., 30, 15-16, (1959).

22 J. D. Huba, H. P. Warren, G. Joyce, X. Pi, B. Iijima, and C. Coker, Global response of the lowlatitude to midlatitude ionosphere due to the Bastille Day flare, Geophys. Res. Lett., 32, L15103, doi:10.1029/2005GL023291 (2005).

23 V. A. Krasnopolsky, Mars' upper atmosphere and ionosphere at low, medium, and high solar activities: Implications for evolution of water, $J$. Geophys. Res., 107, E12, 5128, doi:10.1029/2001JE001809 (2002).

24 E. Kudeki, M. A. Milla, J. L. Chau, L. condori, P. M. Reyes, R. Ilma, D. L. Hysell, and G. Lehmacher, Preliminary results of "World Month" observations at Jicamarca, AGU Fall Meeting, Abstract \#SA43A-1102, 2005.

25 J. Lilensten, W. Kofman, J. Wisemberg, E. S. Oran, and C. R. Devore, Ionization efficiency due to primary and secondary photoelectrons: A numerical model, Ann. Geophys., 7, 83-90 (1989).

24 A. Martinis, R., J. K. Wilson, and M. Mendillo, Modeling day-to-day ionospheric variability on Mars, J. Geophys. Res., 108, A10, 1383, doi:10.1029/2003JA009973 (2003).

25 A. Matsoukas, M. D. Papagiannis, J. Aarons, and J. Klobuchar, Correlation of Solar radio bursts and sudden increases of the total electron content (Sitec) of the ionosphere, J. Atmos. Terr. Phys., 34, 1276-1283 (1972).

26 M. Mendillo et al. (14 co-authors), Behavior of the ionospheric F-region during the great solar flare of August 7, 1972, J. Geophys. Res., 79, 665672 (1974).

27 M. Mendillo and J. V. Evans, Incoherent scatter observations of the ionospheric response to a large solar flare, Radio Sci., 9(2), 197-203 (1974).

28 M. Mendillo, S. M. Smith, J. K. Wilson, H. Rishbeth, and D. P. Hinson, Simultaneous ionospheric variability on Earth and Mars, $J$. Geophys. Res., 108, doi:10.1029/ 2003JA009961 (2003)

29 M. Mendillo, P. Withers, D. Hinson, H. Rishbeth, and B. Reinisch, Effects of solar flares on the ionosphere of Mars, Science, 311, 1135-1138 (2006).

30 P. Mitra, Ionospheric Effects of Solar Flares, D. Reidel, Norwell, MA, 1974.

31 L. E. Moore,., M. Mendillo, I.C.F. MuellerWodarg, and D. L. Murr, Modeling of global variations and ring shadowing in Saturn's ionosphere, Icarus, 172, 503-520 (2004).

32 L. Moore, M. Mendillo, C. Martinis, and S. Bailey, Day-to-day variability of the E-layer, $J$. Geophys. Res., 111, A06307, soi"10.1029/2005JA011448 (2006).

33 M. D. Papagiannis and D.A. Matsoukas, Simultaneous sudden frequency deviations and sudden enhancements of the total electron content of the ionosphere, Nature, 233 (38), 55-56 (1971).

34 J. A. Ratcliffe (ed.), Physics of the Upper Atmosphere, Academic Press, New York, 1960.

35 P. G. Richards and D. G. Torr, Ratios of photoelectron to EUV ionization rates for 
aeronomic studies, J. Geophys. Res., 93, 40604066 (1988).

36 D. Richmond and S.V. Venkateswaran, Geomagnetic crochets and associated ionospheric current systems, Radio Sci., 6, 139-164 (1971).

$37 \mathrm{H}$. Rishbeth and O. K. Garriott, Introduction to Ionospheric Physics, Academic Press, New York, 1969.

38 H. Rishbeth and M. Mendillo, Ionospheric layers of Mars and Earth, Planet. Space Sci., 52, 849-852 (2004).

39 T. Sato, The response of the lower ionosphere to the great solar flare of August 7, 1972, J. Geomag. Geoelectr., 27, 383-407 (1975).

40 R. Schunk and A. Nagy, Ionospheres: Physics, Plasma Physics, and Chemistry, Camb. Univ. Press., NY, 2000.

41 N. R. Thomson, Cr. J. Rodger, and R. L. Dowden, Ionosphere gives size of greatest solar flare, Geophys. Res. Lett., 31, L06803, doi:10.1029/2003GL019345 (2004).

$42 \mathrm{~J}$. E. Titheridge, Direct allowance for the effect of photoelectrons in ionospheric modeling, $J$. Geophys. Res., 101, 357-369 (1996).

43 J. E. Titheridge, Modelling the peak of the ionospheric E-layer, J. Atmos. Solar-Terr. Phys., 62, 93-114 (2000).

44 W. K. Tobiska et al., The SOLAR2000 empirical solar irradiance model and forecast tool, J. Atmos. Solar-Terr. Phys., 62, 1233-1250 (2000).

$45 \mathrm{~W}$. K. Tobiska, SOLAR2000 irradiances for climate change research, aeronomy, space systems engineering, Adv. Space Res., 34, 1736-1746, (2004)
46 T. Tsugawa, S.-R. Zhang, A. J. Coster, Y. Otsuka, J. Sato, A. Saito, Y. Zhang, L. J. Paxton Summerwinter hemisphere asymmetry of the sudden increase in ionospheric total electron content and of the O/N2 ratio: Solar activity dependence, $J$. Geophys. Res., 112, A08301, doi:10.1029/ 2007JA012415 (2007). 